\title{
High Energy Resolution Fluorescence Detection X-Ray Absorption Spectroscopy: Detection of Adsorption Sites in Supported Metal Catalysts
}

\author{
Moniek Tromp ${ }^{1 *}$, Jeroen A. van Bokhoven ${ }^{2}$, Olga V. Safonova ${ }^{3}$, \\ Frank M. F. De Groot ${ }^{4}$, John Evans ${ }^{1,5}$, Pieter Glatzel ${ }^{3}$ \\ ${ }^{1}$ University of Southampton, School of Chemistry, Highfield, Southampton, SO17 1BJ, United Kingdom, \\ ${ }^{2}$ Institute for chemical and bioengineering ETH Zurich, Switzerland, ${ }^{3}$ ESRF, Grenoble, France, \\ ${ }^{4}$ Utrecht University, Utrecht, The Netherland, ${ }^{5}$ Diamond Light Source, Didcot, United Kingdom
}

\begin{abstract}
High energy resolution fluorescence detection (HERFD) X-ray adsorption spectroscopy (XAS) is demonstrated as a new tool to identify the geometry of metal adsorption sites and the orbitals involved in bonding. The type of $\mathrm{CO}$ adsorption site on a nanoparticular $\mathrm{Pt}_{-} \mathrm{Al}_{2} \mathrm{O}_{3}$ catalyst is determined. The orbitals involved in the $\mathrm{Pt}-\mathrm{CO}$ bonding are identified using theoretical FEFF8.0 calculations. In situ application of HERFD XAS is applicable to a large number of catalytic systems and will provide fundamental insights in structure - performance relationships.
\end{abstract}

Keywords: High Energy Resolution, HERFD XAS, XANES, in situ, CO adsorption.

PACS: 82.65.-s

\section{INTRODUCTION}

Optimization of catalytic systems requires a fundamental and detailed understanding of the processes taking place at the catalyst. For heterogeneous catalysts this means the determination of the structures of the catalytically active sites and species. Despite years of research, these structures remain often unknown.

The sensitivity of Pt L edge X-ray Absorption Near Edge Structure (XANES) data for adsorbates on platinum nanoparticles has been reported [1]. The geometry of hydrogen and oxygen adsorption sites was determined by taking difference spectra, i.e. [XANES(Pt cluster + adsorbate) - XANES(Pt cluster bare)], and comparing experimental difference spectra with theoretical ones [1]. The differences in signatures for different adsorption site geometries as such obtained are small and often difficult to interpret.

To detect the subtle variations in XANES upon adsorption of molecules more accurately, spectra with a higher resolution are required. The experimental spectra are broadened because of the finite lifetime of the core hole, resulting in experimental spectra being much broader than the actual density of states (DOS) which is being probed. Monitoring only one fluorescence line with an energy resolution better than the core hole life time broadening results in high energy-resolution fluorescence detection (HERFD) XANES spectra with a much better resolved fine structure, compared to normal transmission or total fluorescence spectra [2,3].

Here we describe the application of HERFD x-ray absorption spectroscopy for the study of different $\mathrm{CO}$ bonding sites structures on platinum nanoparticles. Spectral signatures are related to variations in DOS of the system using the FEFF8 code [4].

\section{EXPERIMENTAL AND THEORY}

The 5 wt $\%$ Pt sample supported on $\gamma-\mathrm{Al}_{2} \mathrm{O}_{3}$ (Condea, 125-250 $\mu \mathrm{m}$ ) was prepared using depositionprecipitation with platinum tetra ammonium nitrate solution in water. The solid was dried and calcined in air $/ \mathrm{N}_{2}$ flow in the following regime: $5 \mathrm{~K} / \mathrm{min}$ to $393 \mathrm{~K}$; $4 \mathrm{~h}$ at $393 \mathrm{~K} ; 5 \mathrm{~K} / \mathrm{min}$ to $673 \mathrm{~K} ; 4 \mathrm{~h}$ at $673 \mathrm{~K}$. Scanning transmission electron microscopy (STEM) shows small platinum particles between 0.5 and $3 \mathrm{~nm}$. The fraction of powder containing 90-125 $\mu$ m particles were separated and introduced into the in situ reactor for X-ray absorption spectroscopic measurements. The gas atmosphere in the reactor was controlled by five mass flow controllers (Bronkhorst). Highly pure

CP882, X-ray Absorption Fine Structure-XAFS13

edited by B. Hedman and P. Pianetta

(C) 2007 American Institute of Physics 978-0-7354-0384-0/07/\$23.00 
$\mathrm{He}, \mathrm{H}_{2}$, and $1 \% \mathrm{CO}$ in $\mathrm{He}$ (Air Liquide) were used as the initial gas mixtures. After reduction of the catalyst in $\mathrm{H}_{2}$ at $473 \mathrm{~K}$ the reactor was purged with $\mathrm{He}$ and cooled down to room temperature. The catalyst was then put in contact with different gas mixtures under constant flux $(10 \mathrm{ml} / \mathrm{min})$ at $298 \mathrm{~K}$. The studies were performed at the high brilliance XAUS-XES beamline ID26 at the European Synchrotron Radiation Facility (ESRF) in Grenoble, France. The electron energy was $6.0 \mathrm{GeV}$ and the ring current varied between 50 and 90 $\mathrm{mA}$. The measurements were performed using two U35 undulators using the $3^{\text {rd }}$ harmonic. The incident energy was selected by means of a pair of liquid nitrogen cooled $\mathrm{Si}(220)$ crystals with an energy bandwidth of $0.7 \mathrm{eV}$. Higher harmonics were suppressed by two mirrors with Cr coating operating in total reflection at $3 \mathrm{mrad}$. The $\mathrm{X}$-ray beam measured $0.3 \mathrm{~mm}$ horizontal and $1 \mathrm{~mm}$ vertical with a total flux on the order of $5^{*} 10^{12}$ photons $/ \mathrm{sec}$ on the sample. The energy was calibrated using a platinum foil. The HERFD was performed with a horizontal plane Rowland circle spectrometer tuned to the Pt $\mathrm{L} \alpha_{1}(9442$ $\mathrm{eV})$ fluorescence line. An energy bandwidth of $1.0 \mathrm{eV}$ in the emission detection was achieved using the (660) Bragg reflection of one spherically-bent Ge wafer. An avalanche photodiode (APD) was used as a detector. A Canberra silicon photodiode was mounted to measure the total fluorescence simultaneously.

FEFF8.2 [4] full multiple scattering, self consistent field, calculations were performed using a HedinLundqvist potential and applying the NOHOLE card. The reduction of the core hole lifetime was achieved by using the EXCHANGE card with parameters 00 1.6.

\section{RESULTS AND DISCUSSION}

Figure 1 compares the Pt foil XANES spectra obtained at ambient conditions using total fluorescence and HERFD detection, obtained simultaneously. It clearly shows the enhancement in spectral resolution of applying the HERFD technique; the XANES features are more pronounced and better resolved.

Similar enhancements in spectral resolution and consequently XANES information provided, is obtained for a $5 \mathrm{wt} \% \mathrm{Pt} / \mathrm{Al}_{2} \mathrm{O}_{3}$ catalyst. In Figure 2 the total fluorescence and HERFD XANES spectra for the reduced sample in $\mathrm{He}$ at room temperature are displayed. Upon changing the gas atmosphere over the catalyst bed to $1 \% \mathrm{CO}$ in $\mathrm{He}$, a significant change in $\mathrm{Pt}$ $\mathrm{L}_{3}$ whiteline shape is observed. For total fluorescence detection, the $\mathrm{CO}$ spectrum shows a shift in energy and an increased whiteline intensity, broadened at higher energies. The spectrum under $\mathrm{CO}$ obtained in HERFD mode displays the energy shift and now reveals a double whiteline feature.

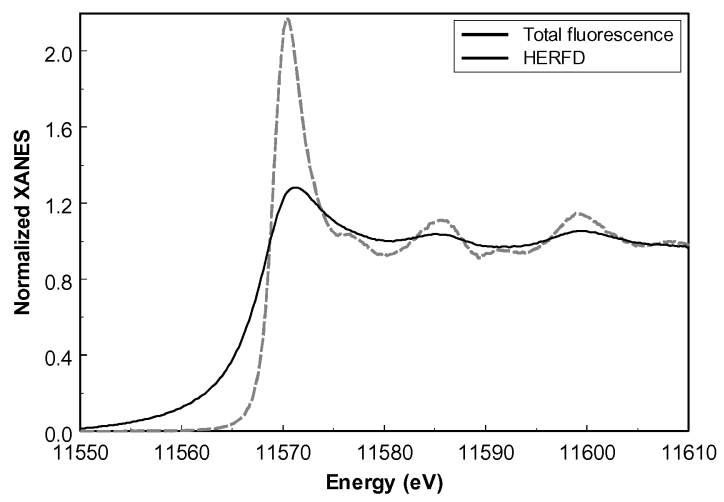

FIGURE 1. Pt $\mathrm{L}_{3}$-edge normalized XANES data for a Pt foil obtained in total fluorescence and HERFD mode.

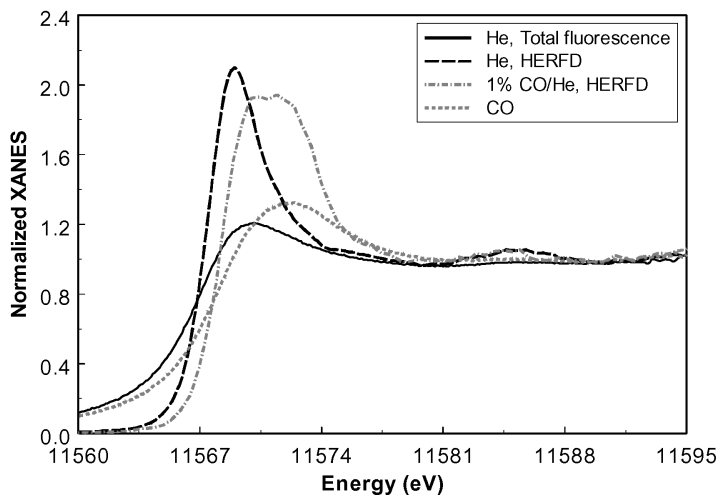

FIGURE 2. $\mathrm{Pt} \mathrm{L}_{3}$ edge normalized XANES data for $5 \mathrm{wt} \%$ $\mathrm{Pt}-\mathrm{Al}_{2} \mathrm{O}_{3}$ in $\mathrm{He}$, obtained in total fluorescence and HERFD mode, and in $1 \% \mathrm{CO} / \mathrm{He}$ in HERFD mode.

FEFF8.0 calculations are performed in order to identify the geometry of the $\mathrm{CO}$ adsorption site. Three different $\mathrm{CO}$ adsorption sites on a $\mathrm{Pt}_{6}[\mathrm{~b}$ ] cluster were considered: $\mathrm{CO}$ atop, $\mathrm{CO}$ bridged and $\mathrm{CO}$ facebridging, Figure 3. Whereas total fluorescence XAFS data is accurately calculated with FEFF without using additional experimental resolution corrections, a reduced life time broadening can be simulated using the EXCHANGE CARD. The HERFD XANES data were fitted using an experimental broadening value $E_{i}$ of $-1.6 \mathrm{eV}$, giving a good approximation to the experiment. 


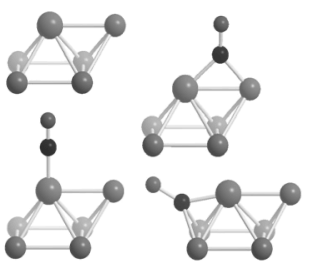

FIGURE 3. $\mathrm{Pt}_{6}-\mathrm{CO}$ cluster simulated with FEFF8.2 (clockwise starting from top left): bare $\mathrm{Pt}_{6}, \mathrm{Pt}_{6}-\mathrm{CO}$ bridged, $\mathrm{Pt}_{6}-\mathrm{CO}$ face bridged, $\mathrm{Pt}_{6}-\mathrm{CO}$ atop.

The theoretical HERFD XANES data of $\mathrm{CO}$ on a $\mathrm{Pt}_{6}$ cluster are presented in Figure 4. The differences in XANES shape between the bridged and face-bridged bonded $\mathrm{CO}$ are very small. The HERFD simulation of the atop $\mathrm{CO}$, however, is very different and shows a double feature in the whiteline.

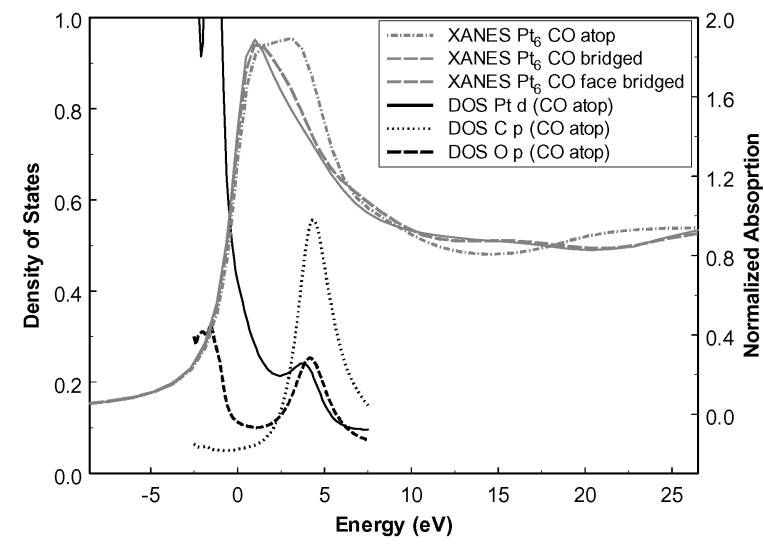

FIGURE 4. Theoretical HERFD Pt $\mathrm{L}_{3}$ edge XANES for the different $\mathrm{CO}$ adsorption sites on $\mathrm{Pt}_{6}$ and the DOS for the $\mathrm{Pt}_{6}$ $\mathrm{CO}$ atop cluster (Fermi level is calculated at $0 \mathrm{eV}$ ).

Comparing the FEFF8.0 (Figure 4) results with the experimental data in $1 \% \mathrm{CO} / \mathrm{He}$ (Figure 2) indicate that the geometry of $\mathrm{CO}$ on Pt nanoparticles (under these experimental conditions) is predominantly atop [5]. This distinction is much easier to assign using the HERFD XANES data.

The DOS of the atop CO adsorption site geometry is given in Figure 4. It is evident that the double feature in the $\mathrm{Pt}_{3}$ edge whiteline originates from Pt d orbital overlap with $\mathrm{C} \mathrm{p}$ and $\mathrm{O} p$ orbitals. This molecular orbital originates from hybridization of $\mathrm{Pt} d$ to the anti-bonding $2 \pi^{*} \mathrm{C}$ and $\mathrm{O}$ orbitals. This orbital is only observed for the atop CO since in this conformation the geometry of the orbitals is most favorable for mixing.

\section{CONCLUSIONS}

HERFD XAS spectroscopy directly probes the structures of adsorption sites on supported metal nanoparticles. Combined with theoretical FEFF calculations the molecular orbitals involved in metaladsorbate bonding can be identified. Hence, HERFD XAS spectroscopy is a promising technique for the in situ characterization of bonding sites of reactants and intermediates, providing fundamental information on reaction mechanisms and allowing structureperformance relations to be derived.

\section{ACKNOWLEDGMENTS}

ESRF for provision of facilities at ID26 and ID24 (MS); financial support (EPSRC to MT, Swiss National Science Foundation to JAvB). Dr. M. Sikora for help in preparing the HERFD experiment.

\section{REFERENCES}

1. (a) M. K. Oudenhuijzen, J. A. van Bokhoven, J. T. Miller, D. E. Ramaker, D. C. Koningsberger, J. Am. Chem. Soc. 127, 1530 (2005); (b) D. E. Ramaker, M. Teliska, Y. Zhang, A. Stakeev, D.C. Koningsberger, Phys. Chem. Chem. Phys. 5, 4492 (2003); (c) A. L. Ankudinov, J. J. Rehr, J. J. Low, S. R. Bare, Topics in Catal. 18, 3 (2002).

2. K. Hämäläinen, D. P. Siddons, J. B. Hastings, L. E. Berman, Phys. Rev. Lett. 67, 2850 (1991).

3. F. M. F. de Groot, M. H. Krish, J. Vogel, J. Phys. Rev. B 66, 195112 (2002).

4. A. L. Ankudinov, C. Bouldin, J. J. Rehr, J. Sims, H. Hung, Phys. Rev. B 65, 104107 (2002).

5. O. Safonova, M. Tromp, J.A. van Bokhoven, F. M. F. de Groot, J. Evans, P. Glatzel, J. Phys. Chem. B, under review (2006). 